Pacific Journal of Mathematics

ON THE REDUCTION OF CERTAIN DEGENERATE PRINCIPAL
SERIES REPRESENATIONS OF SP( $\boldsymbol{n}, C)$ 


\title{
ON THE REDUCTION OF CERTAIN DEGENERATE PRINCIPAL SERIES REPRESENTATIONS $\mathrm{OF} \operatorname{SP}(n, C)$
}

\author{
THOMAS A. FARMER
}

This paper has its origins in the problem of proving irreducibility or reducibility for principal series representations of certain noncompact, complex, semi-simple groups by Fourier-analytic methods; for example, the abelian methods of Gelfand-Naimark for Sl $(n, C)$, and the non commutative (nilpotent) methods of $K$. Gross for $\operatorname{Sp}(n, C)$. As is wellknown, principal series representations are induced from unitary characters of a parabolic subgroup, the series being termed "nondegenerate" if the parabolic is minimal (i.e., the Borel subgroup) and otherwise "degenerate". Here we consider degenerate principal series for $\operatorname{Sp}(n, C)$ corresponding to maximal parabolic subgroups (more general than the situation studied by Gross) and reduce them with respect to the "opposite" parabolic. Let $n_{1}$ denote the complex dimension of the isotropic subspace corresponding to the maximal parabolic, let $0<n_{1}<n$, and $n_{0}=n-n_{1}$. The resulting reduction is described in terms of the natural representation of the complex orthogonal group $O\left(n_{1}, C\right)$ acting on the space $L^{2}\left(C^{n_{1} \times n_{0}}\right)$ and the tensor product of $n_{1}$ copies of the oscillator representation of $\mathrm{Sp}\left(n_{0}, C\right)$. In the terminology introduced by $R$. Howe, this harmonic analysis reduces to the theory of a "dual reductive pair", and any further resolution of the question of irreducibility by these methods will depend upon the study of the oscillator representations for such a dual reductive pair.

We now describe our work in more detail. As a presentation of the complex symplectic group, take

$$
\sum_{n}=\left\{g \in C^{2 n \times 2 n}: g M_{n} g^{\prime}=M_{n}\right\},
$$

where $M_{n}=\left[\begin{array}{rr}0 & -I_{n} \\ I_{n} & 0\end{array}\right], \quad I_{n}$ is the $n \times n$ identity matrix, and $g^{\prime}$ denotes the transpose of $g$. Specify a complete set of conjugacy class representatives of the maximal parabolic subgroups $H$ in $\Sigma_{n}$ (c.f., [9], §8) by defining $H=Z^{\prime} S A$, where the subgroups $Z, S$, and $A$ are given below. Let the isotropic subspace of $C^{2 n}$ corresponding to $H$ have dimension $n_{1}$, with $0<n_{1} \leqq n$ and $n_{0}=n-n_{1}$. Then the blocking scheme used in defining $Z, S$, and $A$ has diagonal blocks of dimensions $n_{1} \times n_{1}, n_{0} \times n_{0}, n_{1} \times n_{1}$, and $n_{0} \times n_{0}$ from upper left to lower right. 


$$
\begin{aligned}
& Z=\left\{\left|\begin{array}{rrrr}
I & 0 & 0 & 0 \\
-y^{\prime} & I & 0 & 0 \\
\eta & x & I & y \\
x^{\prime} & 0 & 0 & I
\end{array}\right|: \eta-\eta^{\prime}=y x^{\prime}-x y^{\prime}\right\} \\
& S=\left\{\left|\begin{array}{llll}
I & 0 & 0 & 0 \\
0 & s_{11} & 0 & s_{12} \\
0 & 0 & I & 0 \\
0 & s_{21} & 0 & s_{22}
\end{array}\right|: s=\left|\begin{array}{ll}
s_{11} & s_{12} \\
s_{21} & s_{22}
\end{array}\right| \in \Sigma_{n_{0}}\right\} \\
& A=\left\{\left|\begin{array}{llll}
\alpha & 0 & 0 & 0 \\
0 & I & 0 & 0 \\
0 & 0 & \alpha^{\prime-1} & 0 \\
0 & 0 & 0 & I
\end{array}\right|: \alpha \in \mathrm{Gl}\left(n_{1}, C\right)\right\}
\end{aligned}
$$

Clearly, $S$ is isomorphic to $\mathrm{Sp}\left(n_{0}, C\right), A$ is isomorphic to $\mathrm{GI}\left(n_{1}, C\right)$ and elements of $S$ commute with elements of $A$. Also, it is easily shown that $Z$ and $Z^{\prime}$ are normalized by $S A$ and hence, $Z S A$ and $Z^{\prime} S A$ are semidirect products.

The maximal parabolic subgroup $H=Z^{\prime} S A$ gives rise to a degenerate principal series of representations $T_{\chi}$ of $\Sigma_{n}$ induced from unitary characters $\chi$ on $H$. We shall realize $T_{\chi}$ in the Hilbert space $L^{2}(Z)$ as follows: Let $d z$ denote Haar measure on the unimodular group $Z$. Denote by $d_{l} h$ and $d_{r} h$, respectively, fixed left and right Haar measures on $H$, and let $\delta_{H}$ be the modular function defined by $\delta_{H}(h)=d_{l} h / d_{r} h$. By direct calculation (cf., [3], §6), $H Z$ is an open subset of $\Sigma_{n}$ whose complement is a set of Haar measure zero. Thus, we can extend the positive character $\delta_{I I}$ and any unitary character $\chi$ on $H$ to functions defined almost everywhere on $\Sigma_{n}$ by defining $\delta_{H}(h z)=\delta_{I I}(h)$ and $\chi(h z)=\chi(h)$ for any $h z \in H Z$. Also, each right coset of $H$ in $\Sigma_{n}$, except for a set of cosets whose union is a null set, contains a unique element of $Z$. It follows that the canonical action of $\Sigma_{n}$ on the right coset space $H \backslash \Sigma_{n}$ gives rise to an "action" of $\Sigma_{n}$ on $Z$ : for any $g \in \Sigma_{n}$ and $z \in Z$, let $z \bar{g}$ be the unique element of $Z$ such that $H(z \bar{g})=H z g$, provided that such an element exists. To be specific, denote by $Z^{g}$ the subset of $Z$ such that $z \bar{g}$ exists, then $Z^{g}$ is an open subset of $Z$ whose complement is a null set. Therefore, if $f \in L^{2}(Z)$ then the function $z \rightarrow f(z \bar{g})$, for fixed $g \in \Sigma_{n}$, is defined almost everywhere in $Z$. Now, the formula ([2], §30) defining the (continuous) unitary representations $T_{\chi}$ of $\Sigma_{n}$, which form a (degenerate) principal series is

$$
T_{\chi}(g) f(z)=\delta_{I I}(z g)^{-1 / 2} \chi(z g) f(z \bar{g}) \quad\left(g \in \Sigma_{n}, f \in L^{2}(Z)\right) .
$$


Let us briefly explain the Fourier-analytic reduction of the restriction of $T_{\chi}$ to the (opposite) parabolic subgroup $Z S A$. Fix $n_{1}$ with $1 \leqq n_{1}<n$, fix $\chi$, and let $T=T_{x}$. We observe that the restriction $\left.T\right|_{Z}$ of $T$ to $Z$ is just the right regular representation of $Z$. Thus, it is natural to replace $T$ with the unitarily equivalent representation $\hat{T}=\mathscr{P} T \mathscr{P}^{-1}$ where $\mathscr{P}$ is the Plancherel transform of $L^{2}(Z)$, for $\left.\hat{T}\right|_{z}$ decomposes as a direct integral. The operator $\mathscr{P}$ maps $L^{2}(Z)$ unitarily onto the Hibert space $L^{2}(\Lambda, X, d m(\lambda))$, of $X$-valued, square-integrable functions on $\Lambda$. Here $\Lambda$ is the dual object of $Z$, $d m(\lambda)$ is the Plancherel measure on $\Lambda$, and $X=H S\left(L^{2}\left(C^{n_{1} \times n_{0}}\right)\right)$ is the Hilbert space of Hilbert-Schmidt operators on $L^{2}\left(C^{n_{1} \times n_{0}}\right)$. It is also the case that $\left.\hat{T}\right|_{S}$ decomposes as a direct integral, and one can explicitly analyze $\hat{T}(z s \alpha)$ for all $z s \alpha \in Z S A$. The operators of $\left.\hat{T}\right|_{z S A}$ involve a representation $\widetilde{I}: S \rightarrow \mathscr{U}\left(L^{2}\left(C^{n_{1} \times n_{0}}\right)\right)$ which is the tensor product of $n_{1}$ copies of the oscillator representation of $\operatorname{Sp}\left(n_{0}, C\right)$, as well as a representation $D: A \rightarrow \mathscr{W}\left(L^{2}\left(C^{n_{1} \times n_{0}}\right)\right)$, in which $\mathrm{Gl}\left(n_{1}, C\right)$ acts on $L^{2}\left(C^{n_{1} \times n_{0}}\right)$ by generalized dilations. The above results are contained in $\S 2$ of this paper.

Let $\mathscr{A}^{\prime}\left(\left.\hat{T}\right|_{Z S A}\right)$ denote the commuting algebra of $\left.\hat{T}\right|_{Z S A}$. There are sufficiently many operators of $\left.\hat{T}\right|_{z S_{A}}$ which are diagonalizable to force $\mathscr{A}^{\prime}\left(\left.\widehat{T}\right|_{Z S_{A}}\right)$ to be decomposable. Moreover, the components of . $\mathscr{A}^{\prime}\left(\left.\widehat{T}\right|_{Z S A}\right)$ are essentially copies of the intersection, $\mathscr{\mathscr { A }}^{\prime}(\widetilde{I}) \cap \mathscr{L}^{\prime}\left(\left.D\right|_{A_{1}}\right)$ of the commuting algebras of $\widetilde{I}$ and $\left.D\right|_{A_{1}}$ where $A_{1} \cong O\left(n_{1}, C\right)$. That is, there is an isometric isomorphism of von Neumann algebras, which we exhibit, between $\mathscr{A}^{\prime}\left(\left.\widehat{T}\right|_{Z S A}\right)$ and $\mathscr{A}^{\prime}(\widetilde{I}) \cap \mathscr{A}^{\prime}\left(\left.D\right|_{A_{1}}\right)$. This is the content of $\S 3$.

It should be noted that there are two special cases in which complete results are known. The case $n_{1}=n$ is special since $Z$ is abelian. It is not difficult to show that $\mathscr{A}^{\prime}\left(\left.\hat{T}\right|_{Z S_{A}}\right)$ is one-dimensional and, hence, for all $\chi, T_{\chi}$ is already irreducible upon restriction to $Z S A$. Also, the irreducibility problem has been completely solved in the case $n_{1}=1, n_{0}=n-1$, in [3], which may be regarded as the prototype for the general case. There it is proved that $T_{\chi}$ is irreducible unless $\chi$ is the trivial character on $H$, in which case, $T_{z}$ splits into the sum of two irreducible representations of $\Sigma_{n}$. The complete results of [3] rest on the fact that the commuting algebra $\mathscr{A}^{\prime}(\widetilde{I}) \cap \mathscr{A}^{\prime}\left(\left.D\right|_{A_{1}}\right)$ is just 2-dimensional when $n_{1}=1$. In the general case, this algebra is infinite dimensional and the full analysis of $\hat{T}$ on all of $\Sigma_{n}$ depends upon its explicit description. ${ }^{1}$

The author wishes to thank Steven Gaal and the referee of an earlier version of this paper for advice and helpful criticism.

$1 \mathrm{R}$. Howe's results show that the joint representation of $\operatorname{Sp}\left(n_{0}, C\right) \times O\left(n_{1}, C\right)$ decomposes continuously. It follows that the commuting algebra is infinite dimensional. 
2. The operators $\hat{T}(z s \alpha)$. In order to analyze $\left.\hat{T}\right|_{z S_{A}}$ we need to introduce the dual object of $Z$, the resulting Plancherel transform of $L^{2}(Z)$ and the oscillator representation of $\operatorname{Sp}\left(n_{0}, C\right)$.

Procedures of Kirillov [5] can be applied to the simply connected, nilpotent lie group $Z$ to yield the dual object - the set of equivalence classes of irreducible, unitary representations of $Z$. The results are given below.

Denote the elements of $Z$ by $(x, y, t)$, where $t=\eta-y x^{\prime}$ so that $t$ is symmetric. In this way $Z$ is identified with $V \times V \times \Lambda_{0}$, where $V=C^{n_{1} \times n_{0}}$ and $\Lambda_{0}=\left\{t \in C^{n_{1} \times n_{1}}: t=t^{\prime}\right\}$. Multiplication in $Z$ is now given by

$$
\left(x_{1}, y_{1}, t_{1}\right)\left(x_{2}, y_{2}, t_{2}\right)=\left(x_{1}+x_{2}, y_{1}+y_{2}, t_{1}+t_{2}-x_{1} y_{2}^{\prime}-y_{2} x_{1}^{\prime}\right) .
$$

Also, the center of $Z$ is easily seen to be $\{0\} \times\{0\} \times \Lambda_{0}$ and the Haar measure of the unimodular group $Z$ is real Lebesgue measure $d z=d x d y d t$ on the Euclidean space $V \times V \times \Lambda_{0}$.

For $\lambda \in \Lambda_{0}$ with rank $\lambda=r$, let $C(\lambda) \in O\left(n_{1}, C\right)$ be such that $C(\lambda) \lambda C(\lambda)^{\prime}=\left|\begin{array}{cc}\lambda_{r} & 0 \\ 0 & 0\end{array}\right|$, where $\lambda_{r}$ is a symmetric, invertible $r \times r$ matrix. Also, let $X=\left|\begin{array}{cc}I_{r} & 0 \\ 0 & 0\end{array}\right| C^{n_{1} \times n_{0}}$, thought of as a measure space with real Lebesgue measure. Finally, throughout this paper we shall let $(u \mid v)=\operatorname{Re} \operatorname{tr} u v^{\prime}$ for all $u, v \in C^{p \times q}$.

TheORem 2.1. Every irreducible, unitary representation of $Z$ is unitarily equivalent to $\Pi_{(\alpha, \beta, \lambda)}$ for some choice of $\alpha, \beta \in V$ and $\lambda \in \Lambda_{0}$, where $\Pi_{(\alpha, \beta, \lambda)}$ is defined as follows:

(1) If $\lambda=0$ then $\Pi_{(\alpha, \beta, \lambda)}$ is 1-dimensional and is given by

$$
\Pi_{(\alpha, \beta, 0)}(x, y, t)=\exp 2 \pi i[(\alpha \mid x)+(\beta \mid y)]
$$

for $(x, y, t) \in Z$.

(2) If rank $\lambda=r \neq 0$, then $\Pi_{\{\alpha, \beta, \lambda)}: Z \rightarrow \mathscr{C}_{(}\left(L^{2}(X)\right)$ is $\infty$-dimensional and is given by

$$
\begin{aligned}
& \Pi_{(\alpha, \beta, \lambda)}(x, y, t) f(u)=\exp 2 \pi i\left[\left(\alpha\left|C(\lambda)^{\prime}\right| \begin{array}{ll}
0_{r} & 0 \\
0 & I
\end{array} \mid C(\lambda) x\right)\right. \\
& \left.+(\beta \mid y)+\left(\lambda \mid t-2 C(\lambda)^{\prime} u y^{\prime}\right)\right] f\left(u+\left|\begin{array}{cc}
I_{r} & 0 \\
0 & 0
\end{array}\right| C(\lambda) x\right)
\end{aligned}
$$

for $f \in L^{2}(X)$ and $(x, y, t) \in Z$. Moreover $\Pi_{\left(\alpha_{1}, \beta_{1}, \lambda_{1}\right)}$ and $\Pi_{\left(\alpha_{2}, \beta_{2}, \lambda_{2}\right)}$ are unitarily equivalent if and only if $\lambda_{1}=\lambda_{2}, \alpha_{1}=\alpha_{2}+\lambda_{1} a$, and $\beta_{1}=\beta_{2}+\lambda_{1} b$ for some $a, b \in V$.

The Plancherel transform of $L^{2}(Z)$ does not require the entire dual object of $Z$, but only the representations corresponding to 
"maximal orbits". These are the representation $\Pi_{(0,0, \lambda)}$, where $\lambda$ is invertible (i.e., $r=n_{1}$ ). Thus, let $\Lambda=\left\{\lambda \in \Lambda_{0}\right.$ : $\left.\operatorname{rank} \lambda=n_{1}\right\}$ and for $\lambda \in \Lambda$ denote $\Pi_{(0,0,2)}$ by $\hat{\lambda}$, then $\hat{\lambda}$ acts in the Hilbert space $L^{2}(V)$ by the formula

$$
\widehat{\lambda}(x, y, t) f(u)=\exp \left[2 \pi i\left(\lambda \mid t-2 u y^{\prime}\right)\right] f(u+x)
$$

for $f \in L^{2}(V)$ and $z=(x, y, t) \in Z$.

Let $\mathscr{S}=\mathscr{S}(V) \times \mathscr{S}(V) \times \mathscr{S}\left(\Lambda_{0}\right)$, where $\mathscr{S}(V)$ (respectively $\left.\mathscr{S}\left(\Lambda_{0}\right)\right)$ is the vector space of all infinitely differentiable, rapidly decreasing functions with domain $V$ (respectively $\Lambda_{0}$ ). Then $\mathscr{S}$ is a dense subspace of $L^{2}(Z)$ with which we can state and prove the following results concerning the Plancherel transform $\mathscr{P}$ of $L^{2}(Z)$.

Theorem 2.3. (1) The Plancherel measure $m$ on $A$ is given by

$$
d m(\lambda)=2^{2 n_{0} n_{1}+n_{1}\left(n_{1}-1\right)} \cdot|\operatorname{det} \lambda|^{2 n_{0}} d \lambda
$$

where $d \lambda$ is the restriction to $\Lambda$ of the Lebesgue measure on $\Lambda_{0}$. The mapping $f \rightarrow K_{f}$ defined for $f \in \mathscr{S}$ by

$$
K_{f}(x, y, \lambda)=\int_{V} \int_{I_{0}} f(x-y, v, t) \exp \left[2 \pi i\left(\lambda \mid t-2 v y^{\prime}\right)\right] d t d v
$$

extends uniquely to a linear isometry of $L^{2}(Z)$ onto $L^{2}\left(V \times V \times \Lambda_{0}\right.$, $d x d y d m(\lambda))$. This is the function-valued Plancherel transform. (3) The mapping $f \rightarrow \hat{f}$ defined weakly for $f \in \mathscr{S}$ by

$$
\hat{f}(\lambda)=\int_{z} f(z) \hat{\lambda}(z) d z
$$

extends uniquely to an isometry $\mathscr{P}^{3}$ of $L^{2}(Z)$ onto $L^{2}\left(\Lambda, H S\left(L^{2}(V)\right)\right.$, $d m(\lambda)$ ). This is the Plancherel transform of $L^{2}(Z)$.

Proof. A computation shows that $\hat{f}(\lambda)$ is an integral operator with kernel $K_{f}$. The mapping $f \rightarrow K_{f}$ is decomposed as in [3] (1.8) into ordinary, partial Fourier transforms

$$
\begin{aligned}
& \mathscr{F}_{2} f(x, y, \lambda)=\int_{V} f(x, v, \lambda) \exp [-2 \pi i(y \mid v)] d v \\
& \mathscr{F}_{3} f(x, y, \lambda)=2^{-n_{1}\left(n_{1}-1\right) / 2} \int_{\Lambda_{0}} f(x, y, t) \exp [-2 \pi i(\lambda \mid t)] d t
\end{aligned}
$$

$\left(f \in \mathscr{S}\right.$, the factor $c=2^{-n_{1}\left(n_{1}-1\right) / 2}$ makes $\mathscr{F}_{3}$ an isometry) and a transformation $\mathscr{R}: L^{2}\left(V \times V \times \Lambda_{0}, d x d y d \lambda\right) \rightarrow L^{2}\left(V \times V \times \Lambda_{0}, d x d y d m(\lambda)\right)$ given by

$$
\mathscr{R} f(x, y, \lambda)=c f(x-y, 2 \lambda y, \lambda) .
$$


In fact, $K_{f}=\mathscr{R}_{2} \mathscr{F}_{2} \mathscr{F}_{3}^{-1} f$ for all $f \in \mathscr{S}$. Now $d m(\lambda)$ is chosen so that $\mathscr{R}$ is an isometry and hence $f \rightarrow K_{f}$ is an isometry.

To prove (3) and (2), one shows that the mapping $K_{f} \rightarrow \widehat{f}$, defined for $f \in \mathscr{S}$, extends to a linear isometry of $L^{2}\left(V \times V \times \Lambda_{0}, d x d y d m(\lambda)\right)$ onto $L^{2}\left(\Lambda, H S\left(L^{2}(V)\right), d m(\lambda)\right)$.

The tensor product of $n_{1}$ copies of the oscillator representation of $S$ occurs naturally in the present setting. Note that $S$ normalizes $Z$. Specifically, for $s \in S$ and $z=(x, y, t) \in Z$

$$
\begin{gathered}
s z s^{-1}=\left(x s_{22}^{\prime}-y s_{21}^{\prime}, y s_{11}^{\prime}-x s_{12}^{\prime}, t+x s_{22}^{\prime} s_{12} x^{\prime}+y s_{21}^{\prime} s_{11} y^{\prime}\right. \\
\left.-x s_{12}^{\prime} s_{21} y^{\prime}-y s_{21}^{\prime} s_{12} x^{\prime}\right) .
\end{gathered}
$$

Let $\lambda \in \Lambda$ and fix $s \in S$. The mapping $z \rightarrow \hat{\lambda}\left(s z s^{-1}\right)$ is an irreducible, unitary representation of $Z$ acting in $L^{2}(V)$ which agrees with $\hat{\lambda}$ on the center of $Z$. Thus, these two irreducible representations are unitarily equivalent, and so, there is a unitary operator $\tilde{\lambda}(s)$ on $L^{2}(V)$ such that

$$
\hat{\lambda}\left(s z s^{-1}\right)=\tilde{\lambda}(s) \hat{\lambda}(z) \tilde{\lambda}(s)^{-1} \quad(z \in Z) .
$$

For each $s, \check{\lambda}(s)$ is unique up to scalar multiples of absolute value 1 , and, as we will show, $\tilde{\lambda}(s)$ can be normalized so that $s \rightarrow \tilde{\lambda}(s)$ is a unitary representation of $S$ acting in $L^{2}(V)$. Let us now be more explicit.

Identify $S$ with $\Sigma_{n_{0}}$ and define the following subgroups of $\Sigma_{n_{0}}$ using the blocking scheme with two diagonal blocks of size $n_{0} \times n_{0}$ :

$$
\begin{aligned}
& M=\left\{m(b)=\left|\begin{array}{ll}
I & b \\
0 & I
\end{array}\right|: b=b^{\prime}\right\} \\
& L=\left\{l(a)=\left|\begin{array}{cc}
a & 0 \\
0 & a^{\vee}
\end{array}\right|: a \in \operatorname{Gl}\left(n_{0}, C\right), a^{\vee}=\left(a^{\prime}\right)^{-1}\right\} .
\end{aligned}
$$

Also, let $p=\left|\begin{array}{rr}0 & -I \\ I & 0\end{array}\right|$. The set $L \cup M \cup\{p\}$ generates $\Sigma_{n_{0}}$ ([3], p. 404), so to define $\tilde{\lambda}$ on $S$ it is enough to define it on this generating set. by

Definition 2.4. Given $\lambda \in \Lambda$, define $\tilde{\lambda}: L \cup M \cup\{p\} \rightarrow \mathscr{U}\left(L^{2}(V)\right)$

$$
\begin{aligned}
& \tilde{\lambda}(l(a)) f(u)=|\operatorname{det} a|^{n_{1}} f(u a) \quad(l(a) \in L) \\
& \tilde{\lambda}(m(b)) f(u)=\exp [-2 \pi i(\lambda u \mid u b)] f(u) \quad(m(b) \in M) \\
& \tilde{\lambda}(p) f(u)=\gamma(\lambda)|\operatorname{det} 2 \lambda|^{n_{0}} U f(2 \lambda u)
\end{aligned}
$$

where $U$ is the Fourier transform of $L^{2}(V)$ defined for $f \in L^{1}(V)$ by 


$$
U f(u)=\int_{V} f(v) \exp [2 \pi i(u \mid v)] d v
$$

and $\gamma(\lambda)$ is a complex number with modulus 1 , which will be determined in the proof of Theorem 2.5.

TheOREM 2.5. The mapping $\tilde{\lambda}$, defined on $L \cup M \cup\{p\}$ above, extends uniquely to be a continuous unitary representation of $\Sigma_{n_{0}}$ (and hence $S$ ) acting in $L^{2}(V)$ which satisfies

$$
\hat{\lambda}\left(s z s^{-1}\right)=\tilde{\lambda}(s) \hat{\lambda}(z) \widetilde{\lambda}(s)^{-1}
$$

for all $s \in S$ and $z \in Z$.

Proof. It is easy to verify that the restrictions of $\tilde{\lambda}$ to $L, M$, and $L M$ are continuous unitary representations of these groups. Now apply Lemma 1 of [3]. To prove that condition (2). of Lemma 1 is satisfied, we use the following: Observe that $m(I) p m(I)=$ $p m(-I) p$. Let $m=m(I)$, then $m^{-1}=m(-I)$. From the definitions of the operators $\tilde{\lambda}(m)$ and $\tilde{\lambda}(p)$, a computation shows that

$$
\begin{aligned}
& \tilde{\lambda}(m) \tilde{\lambda}(p) \widetilde{\lambda}(m) \hat{\lambda}(z) \tilde{\lambda}(m)^{-1} \tilde{\lambda}(p)^{-1} \tilde{\lambda}(m)^{-1} \\
&=\hat{\lambda}\left(m p m z m^{-1} p^{-1} m^{-1}\right) \\
&=\hat{\lambda}\left(p m^{-1} p z p^{-1} m p^{-1}\right) \\
&=\widetilde{\lambda}(p) \widetilde{\lambda}(m)^{-1} \tilde{\lambda}(p) \hat{\lambda}(z) \widetilde{\lambda}(p)^{-1} \tilde{\lambda}(m) \tilde{\lambda}(p)^{-1}
\end{aligned}
$$

and hence $Y_{1}(\lambda)=\tilde{\lambda}(p)^{-1} \tilde{\lambda}(m) \tilde{\lambda}(p)^{-1} \tilde{\lambda}(m) \tilde{\lambda}(p) \tilde{\lambda}(m) \in \mathscr{A}^{\prime}(\hat{\lambda})$, the commuting algebra of $\hat{\lambda}$. This is true regardless of the value of $\gamma(\lambda)$ with $|\gamma(\lambda)|=1$. Thus, letting

$$
Y_{2}(\lambda)=\left[\gamma(\lambda)^{-1} \widetilde{\lambda}(p)\right]^{-1} \widetilde{\lambda}(m)\left[\gamma(\lambda)^{-1} \widetilde{\lambda}(p)\right]^{-1} \tilde{\lambda}(m)\left[\gamma(\lambda)^{-1} \tilde{\lambda}(p)\right] \tilde{\lambda}(m),
$$

we have $Y_{2}(\lambda)=\gamma(\lambda) Y_{1}(\lambda) \in \mathscr{L}^{\prime}(\hat{\lambda})$. But $\hat{\lambda}$ is irreducible so $\mathscr{A}^{\prime}(\hat{\lambda})$ is 1-dimensional and hence $Y_{2}(\lambda)=c(\lambda) I$ for some unique $c(\lambda) \in C$ with $|c(\lambda)|=1$. Define $\gamma(\lambda)=c(\lambda)$ then $Y_{1}(\lambda)=I$ and it follows that

$$
\tilde{\lambda}(m) \tilde{\lambda}(p) \tilde{\lambda}(m)=\tilde{\lambda}(p) \tilde{\lambda}\left(m^{-1}\right) \tilde{\lambda}(p)
$$

which is condition (2) of Lemma 1 of [3].

Just as the representation $\tilde{\lambda}$ of $S$ arises from interwining operators $\tilde{\lambda}(s)$ between $\hat{\lambda}$ and $z \rightarrow \widehat{\lambda}\left(s z s^{-1}\right)$, a representation $D$ of $A$ arises from intertwining operators $D(\alpha)$ between $\left(\alpha \lambda \alpha^{\prime}\right)^{\wedge}$ and $z \rightarrow$ $\hat{\lambda}\left(\alpha^{-1} z \alpha\right)$. For $\alpha \in A$ and $z=(x, y, t) \in Z$, we have

$$
\alpha^{-1} z \alpha=\left(\alpha^{\prime} x, \alpha^{\prime} y, \alpha^{\prime} t \alpha\right),
$$

and from formula (2.2), the representations $\left(\alpha \lambda \alpha^{\prime}\right)^{\wedge}$ and $z \rightarrow \hat{\lambda}\left(\alpha^{-1} z \alpha\right)$ 
are easily seen to agree on the center of $Z$. Hence, these two irreducible representations of $Z$ are unitarily equivalent and, in fact, the operator $D(\alpha)$ given by

$$
D(\alpha) f(u)=|\operatorname{det} \alpha|^{n_{0}} f\left(\alpha^{\prime} u\right) \quad\left(f \in L^{2}(V)\right)
$$

intertwines them. Also, the fact that

$$
\begin{aligned}
\tilde{\lambda}(s) & D(\alpha)^{-1}\left(\alpha \lambda \alpha^{\prime}\right)^{\wedge}(z) D(\alpha) \tilde{\lambda}(s)^{-1} \\
& =\hat{\lambda}\left(s \alpha^{-1} z \alpha s^{-1}\right)=\hat{\lambda}\left(\alpha^{-1} s z s^{-1} \alpha\right) \\
& =D(\alpha)^{-1}\left(\alpha \lambda \alpha^{\prime}\right)^{\sim}(s)\left(\alpha \lambda \alpha^{\prime}\right)^{\wedge}(z)\left(\alpha \lambda \alpha^{\prime}\right)^{\sim}(s)^{-1} D(\alpha)
\end{aligned}
$$

suggests that $D(\alpha)$ may also intertwine $\tilde{\lambda}$ and $\left(\alpha \lambda \alpha^{\prime}\right)^{-}$and, indeed, this is the case. We summarize these facts in the next theorem.

THEOREM 2.6. The mapping $D: A \rightarrow \mathscr{U}\left(L^{2}(V)\right)$ is a continuous unitary representation of $A$ which satisfies

(1) $D(\alpha)^{-1}\left(\alpha \lambda \alpha^{\prime}\right)^{\wedge}(z) D(\alpha)=\widehat{\lambda}\left(\alpha^{-1} z \alpha\right)$

(2) $D(\alpha) \tilde{\lambda}(s) D(\alpha)^{-1}=\left(\alpha \lambda \alpha^{\prime}\right)^{\sim}(s)$

for all $\lambda \in \Lambda, \alpha \in A, s \in S$, and $z \in Z$.

We omit the proof of the above theorem since it is fairly straightforward (cf., [3], Theorem 2), however, we make the following observation related to the proof. For each invertible symmetric matrix $\lambda$ there exists $\beta \in \operatorname{Gl}\left(n_{1}, C\right)$ such that $\lambda=\beta \beta^{\prime}$. Consequently, the action of $A$ on $A$ defined by $\alpha \cdot \lambda=\alpha \lambda \alpha^{\prime}$ is transitive, and from this follow two important facts: First, the function $\gamma$ defined on $\Lambda$ in the proof of Theorem 2.5 is constant. Secondly, we have the

Corollary. Let $\lambda \in \Lambda$ and let $I \in \Lambda$ denote the $n_{1} \times n_{1}$ identity matrix, then $\tilde{\lambda}$ is unitarily equivalent to $\widetilde{I}$.

View $L^{2}(V)$ as the tensor product $\otimes^{n_{1}} L^{2}\left(C^{n_{0}}\right)$ by defining

$$
f_{1} \otimes f_{2} \otimes \cdots \otimes f_{n_{1}}(u)=f_{1}\left(u_{1}\right) f_{2}\left(u_{2}\right) \cdots f_{n_{1}}\left(u_{n_{1}}\right),
$$

where $u_{i}$ is the $i$ th row of $u$ and $f_{i} \in L^{2}\left(C^{n_{0}}\right)$. By inspection of $\widetilde{I}$ and comparison with Theorem 2 of [3], one sees that $\widetilde{I}$ is a tensor product of $n_{1}$ copies of the oscillator representation $\tilde{I}$ of [3].

We may now compute the operators $\hat{T}\left(z_{0} s \alpha\right)$ for $z_{0} s \alpha \in Z S A$. Recall that the formula (1.1) for $T(g)$ involves the action of $g$ on $Z$ given by $H(z \bar{g})=H z \bar{g}$. If $g=z_{0} s \alpha$ then the action becomes $z \overline{z_{0} s \alpha}=$ $\alpha^{-1} s^{-1} z z_{0} s \alpha$. Also, $\delta_{H}(z g)=\delta_{H}\left(z z_{0} s \alpha\right)=\delta_{H}\left((s \alpha)\left(\alpha^{-1} s^{-1} z z_{0} s \alpha\right)\right)=\delta_{H}(s \alpha)$, since $\delta_{H}(h z)$ is defined to be $\delta_{H}(h)$ for any $h z \in H Z$. Furthermore, $\delta_{H}(s \alpha)=\delta_{H}(\alpha)$ because $S$ has no nontrivial characters. Similarly, $\chi\left(z z_{0} s \alpha\right)=\chi(\alpha)$ for any unitary character $\chi$ on $H$. Thus, (1.1) becomes 


$$
T\left(z_{0} s \alpha\right) f(z)=\delta_{H}(\alpha)^{-1 / 2} \chi(\alpha) f\left(\alpha^{-1} s^{-1} z z_{0} s \alpha\right)
$$

for $f \in L^{2}(Z)$. Now let $f \in L^{1}(Z) \cap L^{2}(Z)$ and $\hat{f}=\mathscr{P} f$, then $\hat{f} \in \mathscr{L}=$ $L^{2}\left(\Lambda, H S\left(L^{2}(V)\right), d m(\lambda)\right)$ and $\mathscr{P}\left(L^{1}(Z) \cap L^{2}(Z)\right)$ forms a dense subspace of $\mathscr{K}$. The next theorem determines the transformed representation $\left.\hat{T}\right|_{Z S A}$.

THEOREM 2.8. For $f \in L^{1}(Z) \cap L^{2}(Z)$ and $z s \alpha \in Z S A$,

$$
\hat{T}(z s \alpha) \hat{f}(\lambda)=\delta_{I I}(\alpha)^{1 / 2} \chi(\alpha) \widetilde{\lambda}(s) D(\alpha) \hat{f}\left(\alpha^{-1} \cdot \lambda\right) D(\alpha)^{-1} \widetilde{\lambda}(s)^{-1} \hat{\lambda}(z)^{-1}
$$

for almost every $\lambda \in \Lambda$.

Proof. For every $\lambda \in A, \quad \hat{T}\left(z_{0} s \alpha\right) \hat{f}(\lambda)=\mathscr{P}^{T}\left(z_{0} s \alpha\right) \mathscr{P}^{-1} \mathscr{P} f(\lambda)=$ $\mathscr{S} T\left(z_{0} s \alpha\right) f(\lambda)=\int_{Z} T\left(z_{0} s \alpha\right) f(z) \hat{\lambda}(z) d z=\int_{Z} \delta_{H}(\alpha)^{-1 / 2} \chi(\alpha) f\left(\alpha^{-1} s^{-1} z z_{0} s \alpha\right) \hat{\lambda}(z) d z$

$(1)=\delta_{H}(\alpha)^{-1 / 2} \chi(\alpha) \int_{Z} f(z) \hat{\lambda}\left(s \alpha z \alpha^{-1} s^{-1} z_{0}^{-1}\right) d\left(s \alpha z \alpha^{-1} s^{-1} z_{0}^{-1}\right)$

( 2$)=\delta_{H}(\alpha)^{-1 / 2} \chi(\alpha) \int_{Z} f(z) \hat{\lambda}\left(s \alpha z \alpha^{-1} s^{-1} z_{0}^{-1}\right) \delta_{H}(\alpha) d z$

$(3)=\delta_{H}(\alpha)^{1 / 2} \chi(\alpha)\left[\int_{Z} f(z) \tilde{\lambda}(s) \hat{\lambda}\left(\alpha z \alpha^{-1}\right) \tilde{\lambda}(s)^{-1} d z\right] \hat{\lambda}\left(z_{0}\right)^{-1}$

$(4)=\delta_{H}(\alpha)^{1 / 2} \chi(\alpha) \tilde{\lambda}(s)\left[\int_{Z} f(z) D(\alpha)\left(\alpha^{-1} \cdot \lambda\right)^{\wedge}(z) D(\alpha)^{-1} d z\right] \tilde{\lambda}(s)^{-1} \hat{\lambda}\left(z_{0}\right)^{-1}$

$$
=\delta_{H}(\alpha)^{1 / 2} \chi(\alpha) \tilde{\lambda}(s) D(\alpha)\left[\int_{Z} f(z)\left(\alpha^{-1} \cdot \lambda\right)^{\wedge}(z) d z\right] D(\alpha)^{-1} \tilde{\lambda}(s)^{-1} \hat{\lambda}\left(z_{0}\right)^{-1},
$$

which gives the theorem. Equation (1) is a change of variables, (2) is the fact $d\left(s \alpha z \alpha^{-1} s^{-1}\right)=\delta_{H}(\alpha) d z$ (cf., [6], II. 7), (3) is an application of Theorem 2.5, and (4) is from Theorem 2.6 (1). The formula in the Theorem is said to hold "almost everywhere" since there may be a null set in $\Lambda$ where the right-hand-side is not in $H S\left(L^{2}(V)\right)$.

3. The commuting algebra of $\left.\hat{T}\right|_{Z S A}$. We seek necessary and sufficient conditions for $B \in \mathscr{P}(\mathscr{C})$, the bounded linear operators on the Hilbert space $\mathscr{H}=L^{2}\left(\Lambda, H S\left(L^{2}(V)\right), d m(\lambda)\right)$, to be in the commuting algebra $\mathscr{A}^{\prime}\left(\left.\hat{T}\right|_{Z S A}\right)$. Suppose $B \in \mathscr{A}^{\prime}\left(\left.\hat{T}\right|_{Z S A}\right)$. Then, in particular, $B$ commutes with $\hat{T}(z)$ and $\hat{T}(s)$ for all $z \in Z$ and $s \in S$. We will first see what conditions on $B$ these facts impose. Then we will obtain additional conditions from the fact that $B$ commutes with $\hat{T}(\alpha)$ for $\alpha \in A$.

Realize $H S\left(L^{2}(V)\right)$ as $L^{2}(V) \bar{\otimes} L^{2}(V)$. From the observation that $\mathscr{K}=L^{2}\left(\Lambda, L^{2}(V) \bar{\otimes} L^{2}(V), d m(\lambda)\right)=\int_{\Lambda} L^{2}(V) \bar{\otimes} L^{2}(V) d m(\lambda)$ is a direct integral of Hilbert spaces, we have the notions of decomposable and diagonalizable operators ([7], I. 3). From Theorem 2.8, it is clear that $\hat{T}(z)$ and $\hat{T}(s)$ are decomposable operators for $z \in Z$ and $s \in S$ and can be denoted: 


$$
\begin{aligned}
& \hat{T}(z)=\int_{1} I \bar{\otimes} \hat{\lambda}(z) d m(\lambda) \\
& \hat{T}(s)=\int_{\Lambda} \tilde{\lambda}(s) \bar{\otimes} \tilde{\lambda}(s) d m(\lambda) .
\end{aligned}
$$

To show from this that $B$ is decomposable requires the following technical lemma of Stone-Weierstrass type.

Let $X$ be a locally compact, $\sigma$-compact, Hausdorff space with positive Borel measure $\mu$, which is finite on compact sets. Let $H$ be a separable Hilbert space. For $a \in L^{\infty}(X, \mu)$, let $M(a)$ denote the diagonalizable operator on $L^{2}(X, H, \mu)$ given by $M(a) f(x)=a(x) f(x)$.

Lemma 3.1. Let $\mathscr{X}$ be a subalgebra of $C(X) \cap L^{\infty}(X, \mu)$ over $C$ such that

(a) $1 \in \mathscr{A}$,

(b) $a \in \mathscr{A}$ implies $\bar{a} \in \mathscr{C}$,

(c) $\mathscr{A}$ separates points of $X$.

If $B \in \mathscr{L}\left(L^{2}(X, H, \mu)\right)$ and $B M(a)=M(a) B$ for all $a \in \mathscr{A}$, then $B M(a)=$ $M(a) B$ for all $a \in L^{\infty}(X, \mu)$.

THEOREM 3.2. Suppose $B \in \mathscr{S}^{\prime}\left(\left.\widehat{T}\right|_{Z S A}\right)$. Then

(a) $B$ is decomposable.

(b) There exists a mapping $\lambda \rightarrow B(\lambda)$ of $\Lambda$ into $\mathscr{L}\left(L^{2}(V)\right)$, defined a.e. $[m]$, such that $B=\int_{A} B(\lambda) \bar{\otimes} \operatorname{Idm}(\lambda)$.

(c) $B(\lambda) \in \mathscr{A}^{\prime}(\tilde{\lambda})$ for almost every $\lambda \in \Lambda$.

Proof. (a) Let $\mathscr{A}=\left\{\lambda \rightarrow \exp [2 \pi i(\lambda \mid t)]: t \in \Lambda_{0}\right\}$. $\mathscr{A}$ is a subalgebra over $C$ of $C(\Lambda) \cap L^{\infty}(\Lambda, m)$, which satisfies the conditions of Lemma 3.1. Furthermore, if $a \in \mathscr{A}$ then the associated diagonalizable operator $M(a) \in \mathscr{L}(\mathscr{H})$, given by $M(a) \hat{f}(\lambda)=a(\lambda) \hat{f}(\lambda)$, is an operator of the representation $\left.\hat{T}\right|_{z}$. In fact, if $a(\lambda)=\exp [2 \pi i(\lambda \mid t)]$ then $M(a)=\widehat{T}(z)$, where $z=(0,0, t)$. Therefore $B M(a)=M(a) B$ for every $a \in \mathscr{A}$ and the lemma implies that this holds for every $a \in$ $L^{\infty}(\Lambda, m)$. Since $\left\{M(a): a \in L^{\infty}(\Lambda, m)\right\}$ is exactly the set of diagonalizable operators on $\mathscr{Y}, B$ must be decomposable ([7], I. 3.2).

(b) Since $B$ is decomposable, there exists an essentially bounded mapping $\lambda \rightarrow B_{\lambda}$, defined a.e. $[m]$ with values in $\mathscr{P}\left(L^{2}(V) \bar{\otimes} L^{2}(V)\right)$, such that $B=\int_{1} B_{\lambda} d m(\lambda)$.

Since $B$ commutes with $\hat{T}(z)=\int_{A} I \bar{\otimes} \hat{\lambda}(z) d m(\lambda)$ for every $z \in Z$, $B_{\lambda}$ commutes with $I \bar{\otimes} \hat{\lambda}(z)$ except for $\lambda$ in an $m$-null set $N_{z}$. $Z$ is separable; let $\left\{z_{i}: i \in \mathscr{F}\right\}$ be a countable dense subset of $Z$ and let $N=\bigcup_{i \in \mathscr{C}} N_{z_{i}}$. Then $m(N)=0$ and for $\lambda \in N^{c}, B_{\lambda}$ commutes with $I \bar{\otimes} \hat{\lambda}\left(z_{i}\right)$ for all $i \in \mathscr{I}$. Thus we have two continuous maps, $z \rightarrow$ 
$B_{\lambda}(I \bar{\otimes} \hat{\lambda}(z))$ and $z \rightarrow(I \bar{\otimes} \hat{\lambda}(z)) B_{\lambda}$, which agree on a dense subset of $Z$. It follows that $B_{\lambda} \in \mathscr{A}^{\prime}(I \bar{\otimes} \hat{\lambda})$ for all $\lambda \in N^{c}$. Since $\hat{\lambda}$ is irreducible, for each $\lambda \in N^{c}$ there exists $B(\lambda) \in \mathscr{L}\left(L^{2}(V)\right)$ such that $B_{\lambda}=B(\lambda) \bar{\otimes} I$ ([1], VI. 3.14). Therefore, $B=\int_{A} B(\lambda) \bar{\otimes} \operatorname{Idm}(\lambda)$.

(c) Since $B$ commutes with $\hat{T}(s)=\int_{A} \tilde{\lambda}(s) \bar{\otimes} \tilde{\lambda}(s) d m(\lambda)$ for every $s \in S, B(\lambda) \bar{\otimes} I$ commutes with $\tilde{\lambda}(s) \bar{\otimes} \tilde{\lambda}(s)$ a.e. $[m]$. Just as in the proof of (b), since $S$ is separable there exists an $m$-null set $N$ such that for every $\lambda \in N^{c}$ and every $s \in S$,

$$
(B(\lambda) \bar{\otimes} I)(\tilde{\lambda}(s) \bar{\otimes} \tilde{\lambda}(s))=(\tilde{\lambda}(s) \bar{\otimes} \tilde{\lambda}(s))(B(\lambda) \bar{\otimes} I) .
$$

It follows that $B(\lambda) \tilde{\lambda}(s) \bar{\otimes} \tilde{\lambda}(s)=\tilde{\lambda}(s) B(\lambda) \bar{\otimes} \tilde{\lambda}(s)$ and hence $B(\lambda) \tilde{\lambda}(s)=$ $\tilde{\lambda}(s) B(\lambda)$. Thus, $B(\lambda) \in \mathscr{L}^{\prime}(\tilde{\lambda})$ for almost every $\lambda$.

Continue to suppose that $B \in \mathscr{A}^{\prime}\left(\left.\hat{T}\right|_{z S A}\right)$ so that $B$ satisfies (a), (b), and (c) of Theorem 3.2. We will now make use of the condition that $B \hat{T}(\alpha)=\widehat{T}(\alpha) B$ for all $\alpha \in A$. Recall that $\alpha$ denotes both an element of $\mathrm{Gl}\left(n_{1}, C\right)$ and the corresponding element $\left|\begin{array}{llll}\alpha & 0 & 0 & 0 \\ 0 & I & 0 & 0 \\ 0 & 0 & \alpha^{\prime-1} & 0 \\ 0 & 0 & 0 & I\end{array}\right|$ of $A$.

Concerning the transitive action of $A$ on $\Lambda$ given by $\alpha \cdot \lambda=\alpha \lambda \alpha^{\prime}$, let $A_{1}$ be the stability subgroup of $A$ at $I \in \Lambda$. That is, $A_{1}=$ $\left\{\alpha \in A: \alpha \alpha^{\prime}=I\right\}$, which can be identified with $O\left(n_{1}, C\right)$. Let $p: A \rightarrow \Lambda$ be the projection $p(\alpha)=\alpha \cdot I=\alpha \alpha^{\prime}$. We will need to know that a measurable set $N$ is an $m$-null set in $\Lambda$ if and only if $p^{-1}(N)$ is a null set in $A$ with respect to Haar measure. This result can be obtained by first showing that $d \eta(\lambda)=|\operatorname{det} \lambda|^{-\left(n_{1}+1\right)} d \lambda$ is an $A$-invariant measure on $A$. It follows (as in [1], V. 3) that $N$ is an $\eta$-null set in $\Lambda$ if and only if $p^{-1}(N)$ is a null set in $A$. Since $m$ and $\eta$ are clearly equivalent we have the needed result. We are now able to prove

THEOREM 3.3. If $B \in \mathscr{A}^{\prime}\left(\left.\widehat{T}\right|_{Z S A}\right)$ then there exists a mapping $\lambda \rightarrow B(\lambda)$ of $\Lambda$ into $\mathscr{L}\left(L^{2}(V)\right)$ which is weakly continuous and satisfies:

(a) $B=\int_{A} B(\lambda) \bar{\otimes} \operatorname{Idm}(\lambda)$

(b) $B\left(\alpha \alpha^{\prime}\right)=D(\alpha) B(I) D(\alpha)^{-1}$ for all $\alpha \in A$;

(c) $B(I) \in \mathscr{A}^{\prime}\left(\left.D\right|_{A_{1}}\right)$;

(d) $B(\lambda) \in \mathscr{A}^{\prime}(\tilde{\lambda})$ for all $\lambda \in \Lambda$.

Proof. From Theorem 3.2, we have a weakly measurable mapping $\lambda \rightarrow B_{1}(\lambda)$ such that $B=\int_{A} B_{1}(\lambda) \bar{\otimes} \operatorname{Idm}(\lambda)$. The major part of 
this proof is to show that there is an equivalent mapping which is weakly continuous. By Theorem 2.8, if $\alpha \in A$ and $\hat{f} \in \mathscr{P}\left(L^{1}(Z) \cap L^{2}(Z)\right)$ then

$$
\widehat{T}(\alpha) \hat{f}(\lambda)=\delta_{H}(\alpha)^{1 / 2} \chi(\alpha) D(\alpha) \hat{f}\left(\alpha^{-1} \cdot \lambda\right) D(\alpha)^{-1}
$$

for almost every $\lambda \in \Lambda$, where $\alpha^{-1} \cdot \lambda=\alpha^{-1} \alpha^{\prime-1}$. The condition that $B \widehat{T}(\alpha)=\widehat{T}(\alpha) B$ for all $\alpha \in A$ is seen to be equivalent to

(1) For all $\alpha \in A, B_{1}(\lambda)=D(\alpha)^{-1} B_{1}\left(\alpha \lambda \alpha^{\prime}\right) D(\alpha)$ a.e. $[m(\lambda)]$.

Consider the weakly measurable mapping $\alpha \rightarrow D(\alpha)^{-1} B_{1}\left(\alpha \alpha^{\prime}\right) D(\alpha)$ of $A$ into $\mathscr{L}\left(L^{2}(V)\right)$. For fixed $\alpha \in A$, (1) implies

$$
D(\alpha \beta)^{-1} B_{1}\left(\alpha \beta \beta^{\prime} \alpha^{\prime}\right) D(\alpha \beta)=D(\beta)^{-1} B_{1}\left(\beta \beta^{\prime}\right) D(\beta) \text { a.e. }[d \beta],
$$

since a null set in $\Lambda$ pulls back under $p^{-1}$ to a null set in $A$. Fix $\phi, \psi \in L(V)$ and define the measurable, essentially bounded function $w: A \rightarrow C$ by

$$
w(\beta)=\left(D(\beta)^{-1} B_{1}\left(\beta \beta^{\prime}\right) D(\beta) \phi, \psi\right),
$$

where (, ) is the inner product of $L(V)$. Then, by $(2), w(\beta) d \beta$ defines a left invariant Borel measure on $A$. By uniqueness of Haar measure, $w$ must be almost everywhere constant. Moreover, if this number is denoted $w_{\phi, \psi}$ then, by application of the Riesz representation theorem to the bilinear form $(\phi, \psi) \rightarrow w_{\phi, \psi}$, there exists a unique $L \in \mathscr{L}\left(L^{2}(V)\right)$ such that

$$
D(\beta)^{-1} B_{1}\left(\beta \beta^{\prime}\right) D(\beta)=L \text { a.e. }[d \beta] .
$$

Consider the weakly measurable maps $\beta \rightarrow B_{1}\left(\beta \beta^{\prime}\right)$ and $\beta \rightarrow$ $D(\beta) L D(\beta)^{-1}$ of $A$ into $\mathscr{L}\left(L^{2}(V)\right)$. Since $A_{1}=\left\{\alpha \in A: \alpha \alpha^{\prime}=I\right\}$, the first map is constant on left cosets of $A_{1}$. The second map is continuous (with respect to either the strong or the weak operator topology of $\mathscr{L}\left(L^{2}(V)\right)$ ). Also, (3) implies that the two maps coincide almost everywhere. We can conclude that $\beta \rightarrow D(\beta) L D(\beta)^{-1}$ is both continuous and constant on left cosets of $A_{1}$. Because of this fact, the mapping $\beta \beta^{\prime} \rightarrow D(\beta) L D(\beta)^{\prime}$ is well-defined on 4 . It is also continuous since $\beta \rightarrow D(\beta) L D(\beta)^{-1}$ is continuous and $p$ is open. Define $B\left(\beta \beta^{\prime}\right)=D(\beta) L D(\beta)^{\prime}$, then $B\left(\beta \beta^{\prime}\right)$ and $B_{1}\left(\beta \beta^{\prime}\right)$ differ only on a "strip" set of measure zero in $A$, which projects to a null set in $\Lambda$. Thus, $\lambda \rightarrow B(\lambda)$ is a continuous mapping such that (a) holds.

Parts (b) and (c) follow immediately from the definition of $B(\lambda)$. To prove (d), recall from Theorem 3.2 (c) that $B(\lambda) \in \mathscr{A}^{\prime}(\tilde{\lambda})$ for almost every $\lambda \in \Lambda$. In particular, $B\left(\lambda_{0}\right) \in \mathscr{A}^{\prime}\left(\tilde{\lambda}_{0}\right)$ for some $\lambda_{0}=$ $\beta \beta^{\prime} \in \Lambda$. Let $\lambda \in \Lambda$, then $\lambda=\alpha \lambda_{0} \alpha^{\prime}=\alpha \beta \beta^{\prime} \alpha^{\prime}$ for some $\alpha \in A$. Now apply the definition of $B(\lambda)$ along with Theorem $2.6(2)$. 
We now have the main theorem.

THEOREM 3.4. The mapping

$$
B(I) \longrightarrow B=\int_{A} D(\beta) B(I) D(\beta)^{-1} \bar{\otimes} \operatorname{Idm}\left(\beta \beta^{\prime}\right)
$$

is an isomorphism of von Neumann algebras from $\mathscr{A}^{\prime}\left(\left.D\right|_{A_{1}}\right) \cap \mathscr{A}^{\prime}(\widetilde{I})$ onto $\mathscr{L}^{\prime}\left(\left.\hat{T}\right|_{Z S A}\right)$.

Proof. The mapping in the theorem makes sense because the condition $B(I) \in \mathscr{A}^{\prime}\left(\left.D\right|_{A_{1}}\right)$ guarantees that $\beta \beta^{\prime} \rightarrow D(\beta) B(I) D(\beta)^{-1} \bar{\otimes} I$ is well-defined. It is straightforward to verify separately that $B \in$ $\mathscr{A}^{\prime}\left(\left.\widehat{T}\right|_{Z}\right), B \in \mathscr{A}^{\prime}\left(\left.\hat{T}\right|_{S}\right)$, and $B \in \mathscr{A}^{\prime}\left(\left.\widehat{T}\right|_{A}\right)$. Also, using properties of decomposable operators, it is easy to show that $B(I) \rightarrow B$ is an isometric, *-algebra isomorphism. The fact that it is surjective is proved in Theorem 3.3.

\section{REFERENCES}

1. S. Gaal, Linear Analysis and Representation Theory, New York, Heidelberg, Berlin, Springer 1973.

2. I. M. Gelfand and M. A. Naimark, Unitäre Darstellungen der Klassichen Gruppen, Berlin, Akadamie 1957.

3. K.I. Gross, The dual of a parabolic subgroup and a degenerate principal series of $\mathrm{Sp}(n, C)$, Amer. J. Math., 93 (1971), 398-428.

4. R. Howe, Remarks on classical invariant theory, preprint.

5. A. A. Kirillov, Unitary representations of nilpotent groups and harmonic analysis on nil and solvmanifolds, Symp. in Pure Math., Providence, AMS 1973.

6. L. Nachbin, The Haar Integral, Princeton, N. J., Van Nostrand 1965.

7. J. T. Schwartz, $W^{*}$-Algebras, London, Nelson 1968.

8. A. Weil, Sur certains groupes d'operateurs unitairies, Acta Math., 111 (1964), 143-211.

9. J.A. Wolf, Unitary representations of maximal parabolic subgroups of the classical groups, Amer. Math. Soc. Memoir, 180 (1976).

Received October 25, 1978.

MIAMI UNIVERSITY

OXFORD, OH 45056 



\section{PACIFIC JOURNAL OF MATHEMATICS}

\section{EDITORS}

DONALD BABBITT (Managing Editor)

University of California

Los Angeles, CA 90024

HUGO RossI

University of Utah

Salt Lake City, UT 84112

C. C. Moore and ANDrew OGG

University of California

Berkeley, CA 94720
J. DUGUNDJI

Department of Mathematics University of Southern California Los Angeles, CA 90007

R. FINN and J. MILGRAM

Stanford University

Stanford, CA 94305

\section{ASSOCIATE EDITORS}
E. F. BeCKENBACH
B. H. NeumanN
F. WOLF
K. YOSHIDA

\section{SUPPORTING INSTITUTIONS}

UNIVERSITY OF BRITISH COLUMBIA CALIFORNIA INSTITUTE OF TECHNOLOGY UNIVERSITY OF CALIFORNIA MONTANA STATE UNIVERSITY UNIVERSITY OF NEVADA, RENO NEW MEXICO STATE UNIVERSITY OREGON STATE UNIVERSITY UNIVERSITY OF OREGON

\author{
UNIVERSITY OF SOUTHERN CALIFORNIA \\ STANFORD UNIVERSITY \\ UNIVERSITY OF HAWAII \\ UNIVERSITY OF TOKYO \\ UNIVERSITY OF UTAH \\ WASHINGTON STATE UNIVERSITY \\ UNIVERSITY OF · WASHINGTON
}

The Supporting Institutions listed above contribute to the cost of publication of this Journal, but they are not owners or publishers and have no responsibility for its content or policies.

Mathematical papers intended for publication in the Pacific Journal of Mathematics should be in typed form or offset-reproduced, (not dittoed), double spaced with large margins. Please do not use built up fractions in the text of the manuscript. However, you may use them in the displayed equations. Underline Greek letters in red, German in green, and script in blue. The first paragraph or two must be capable of being used separately as a synopsis of the entire paper. Please propose a heading for the odd numbered pages of less than 35 characters. Manuscripts, in triplicate, may be sent to any one of the editors. Please classify according to the scheme of Math. Reviews, Index to Vol. 39. Supply name and address of author to whom proofs should be sent. All other communications should be addressed to the managing editor, or Elaine Barth, University of California, Los Angeles, California, 90024.

50 reprints to each author are provided free for each article, only if page charges have been substantially paid. Additional copies may be obtained at cost in multiples of 50 .

The Pacific Journal of Mathematics is issued monthly as of January 1966. Regular subscription rate: $\$ 84.00$ a year (6 Vols., 12 issues). Special rate: $\$ 42.00$ a year to individual members of supporting institutions.

Subscriptions, orders for numbers issued in the last three calendar years, and changes of address should be sent to Pacific Journal of Mathematics, P.O. Box 969, Carmel Valley, CA 93924, U.S.A. Older back numbers obtainable from Kraus Periodicals Co., Route 100, Millwood, NY 10546.

PUBLISHED BY PACIFIC JOURNAL OF MATHEMATICS, A NON-PROFIT CORPORATION

Printed at Kokusai Bunken Insatsusha (International Academic Printing Co., Ltd.). 8-8, 3-chome, Takadanobaba, Shinjuku-ku, Tokyo 160, Japan.

Copyright (C) 1979 by Pacific Journal of Mathematics Manufactured and first issued in Japan 


\section{Pacific Journal of Mathematics}

\section{Vol. 84, No. $2 \quad$ June, 1979}

Somesh Chandra Bagchi and Alladi Sitaram, Spherical mean periodic

functions on semisimple Lie groups ........................ 241

Billy Joe Ball, Quasicompactifications and shape theory............. 251

Maureen A. Bardwell, The o-primitive components of a regular ordered permutation group ................................ 261

Peter W. Bates and James R. Ward, Periodic solutions of higher order

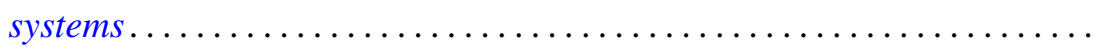

Jeroen Bruijning, A characterization of dimension of topological spaces by totally bounded pseudometrics......................... 283

Thomas Farmer, On the reduction of certain degenerate principal series

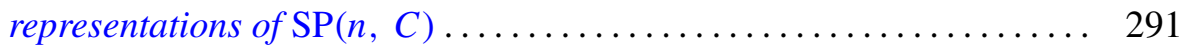

Richard P. Jerrard and Mark D. Meyerson, Homotopy with m-functions . . . . 305

James Edgar Keesling and Sibe Mardesic, A shape fibration with fibers of

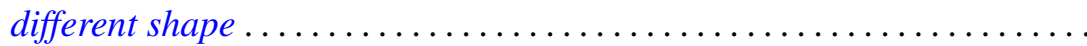

Guy Loupias, Cohomology over Banach crossed products. Application to bounded derivations and crossed homomorphisms ...............

Rainer Löwen, Symmetric planes ........................ 367

Alan L. T. Paterson, Amenable groups for which every topological left

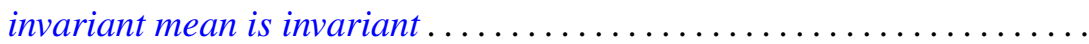

Jack Ray Porter and R. Grant Woods, Ultra-Hausdorff H-closed extensions

Calvin R. Putnam, Operators satisfying a $G_{1}$ condition .

Melvin Gordon Rothenberg and Jonathan David Sondow, Nonlinear smooth representations of compact Lie groups..............

Werner Rupp, Riesz-presentation of additive and $\sigma$-additive set-valued measures.

A. M. Russell, A commutative Banach algebra of functions of generalized variation

Judith D. Sally, Superregular sequences

Patrick Shanahan, On the signature of Grassmannians . . 